\title{
Determinación de rangos de movimiento del miembro superior en una muestra de estudiantes universitarios mexicanos
}

Determination of Range of Motion for Upper Limbs in a Sample of Mexican University Students

\section{Determinação de Rangos de Movimento do Membro Superior em uma Amostra de Estudantes Universitários Mexicanos}

\author{
Laura Esmeralda Peña Ayala, Ing.'; \\ Karla Gabriela Gómez Bull, MI.'; \\ María Marisela Vargas Salgado, Dra..; \\ Gabriel Ibarra Mejía, PhD.2; \\ Aurora Irma Máynez Guaderrama, Dra. ${ }^{1}$
}

Recibido: 19 de junio de 2017 / Aprobado: 7 de noviembre de 2017

Doi: http://dx.doi.org/10.12804/revistas.urosario.edu.co/revsalud/a.6845

Para citar este artículo: Peña-Ayala LE, Gómez-Bull KG, Vargas-Salgado MM, Ibarra-Mejía G, Máynez Guaderrama AI.

Determinación de rangos de movimiento del miembto superior en una muestra de estudiantes universitarios. Rev Cienc Salud.

2018; 16(especial): p. 64-74. Doi: http://dx.doi.org/10.12804/revistas.urosario.edu.co/revsalud/a.6845

\section{Resumen}

Introducción: esta investigación se realizó en la Universidad Autónoma de Ciudad Juárez, en la División Multidisciplinaria de la Ciudad Universitaria, con una muestra de estudiantes del programa de Ingeniería Industrial y de Sistemas. Su objetivo fue crear una base de datos que contuviera información sobre los rangos de movimientos (ROMs) del miembro superior de una muestra de jóvenes estudiantes. Materiales y métodos: se utilizó un inclinómetro de burbuja marca Baseline® y el kit antropométrico Rosscraft®. El diseño de la investigación fue de tipo descriptivo, cuantitativo y transversal, y se tomó una muestra de 50 estudiantes; la determinación de los Roms consistió en medir a cada uno de los participantes para, posteriormente, capturar y analizar los datos a través del software Minitab17®, para determinar

\footnotetext{
1 Universidad Autónoma de Ciudad Juárez.

Autor de correspondencia: karla.gomez@uacj.mx

2 Universidad de Texas en El Paso.
} 
medidas de tendencia central y la existencia de diferencia significativa entre los roms de hombres y mujeres. Resultados: se determinó el límite de rango de movimiento para las articulaciones del codo, hombro, muñeca y antebrazo, encontrando que no existe evidencia de diferencia significativa entre los Roms de los hombres y las mujeres del estudio, a excepción de la abducción en el hombro derecho. Conclusión: los datos recopilados en esta investigación pueden ser utilizados para el diseño de áreas de trabajo que se adecúen a las necesidades del usuario, de forma que se evite la presencia de lesiones músculo-esqueléticas y se logre la reducción de la fatiga física en los trabajadores.

Palabras clave: rangos de movimiento, rango de movimiento articular, ergonomía.

\section{Abstract}

Introduction: This research was carried out at the Universidad Autonoma de Ciudad Juarez, División Multidisciplinaria Ciudad Universitaria, with a sample of students of the Industrial and Systems Engineering Program. With the objective of creating a database containing information about the ranges of motion (Roms) of the upper limb. Materials and Methods: Upper limb Roms estimation was developed using a bubble inclinometer Baseline ${ }^{\circledR}$ and the Rosscraft ${ }^{\circledR}$ anthropometric kit. The design of the research was descriptive, quantitative and transversal. A sample of 50 students was taken; the determination of the Roms consisted in measuring each of the participants and, subsequently, the data were captured and analyzed through Minitab17® software, in order to obtain measures of central tendency and the existence of significant difference between the Roms of men and women. Results: The Range of motion limit for the elbow, shoulder, wrist and forearm joints was determined, finding that there is no evidence of a significant difference between Roms of men and women in the study, with the exception of abduction in the right shoulder. Conclusion: The data collected in this research can be used to design workspaces that fit the needs of the user, with the aim to avoid the presence of musculoskeletal injuries and reduce physical fatigue among workers.

Keywords: range of motion, joint range of motion, ergonomics.

\section{Resumo}

Introdução: Esta pesquisa se realizou na Universidad Autónoma de Ciudad Juárez, Divisão Multidisciplinar Cidade Universitária, com uma amostra de estudantes do programa de Engenharia Industrial e de Sistemas. Com o objetivo de criar uma base de dados que contenha informação sobre os rangos de movimentos (ROMs) do membro superior de uma amostra de jovens estudantes. Materiais e métodos: se utilizou um inclinómetro de borbulha marca Baseline ${ }^{\circledR}$ e o kit antropométrico Rosscraft ${ }^{\circledR}$. O desenho da pesquisa foi de tipo descritivo, quantitativo e transversal, se tomou uma amostra de 50 estudantes; a determinação dos Roms consistiu em medir a cada um dos participantes y posteriormente os dados foram capturados e analisados através do software Minitab17®, para determinar medidas de tendência central e a existência de diferença significativa entre os Roms de homens e mulheres. Resultados: se determinou o limite de rango de movimento para as articulações do cotovelo, ombro, pulso e o antebraço, encontrando que não existe evidência de diferença significativa entre os Roms dos homens e as mulheres do estudo, a excepção da abdução no ombro direito. Conclusão: : os dados compilados nesta pesquisa podem ser utilizados no desenho das áreas de trabalho que se adequem às necessidades do usuário, de forma que se evite a presença de lesões musculoesqueléticas, e consiga-se a redução da fadiga física nos trabalhadores.

Palavras-chave: rangos de movimento, rango de movimento articular, ergonomia. 


\section{Introducción}

$\mathrm{U}_{\mathrm{e}}^{\mathrm{n}}$ no de los campos de aplicación de la ergonomía es la biomecánica, ciencia que estudia el cuerpo humano, sus movimientos y limitaciones, y analiza las destrezas motoras. Esta área ayuda a la interpretación correcta del cuerpo humano para tener un mayor provecho del diseño de producto o estancia de trabajo y así evitar lesiones (1). Por su parte, la ergonomía científica estudia las interacciones entre seres humanos y sistemas, aplicando datos y métodos al diseño, con el objetivo de optimizar el bienestar del ser humano y el desempeño de los sistemas (2). La rama de la biomecánica relacionada con la ergonomía se conoce como biomecánica ocupacional, la cual se encarga del estudio de la interacción física del trabajador con su entorno para mejorar su rendimiento laboral y minimizar riesgos. Esta ciencia estudia el cuerpo desde un enfoque mecánico y desde el punto de vista del aparato locomotor, como el órgano del cuerpo humano encargado de producir movimientos, por lo tanto, el aparato locomotor se puede considerar como una maquina adaptada al movimiento (3).

El rango de movimiento, conocido también como Roм por las siglas de Rank of Movement, es el ángulo máximo descrito entre dos segmentos del cuerpo con un plano de referencia, el cual es realizado por medio de articulaciones (4), es decir, es el número de grados a través del cual una articulación es capaz de moverse. El Rom también es conocido como la flexión de las articulaciones o como el grado de contracción muscular y la deformabilidad de las articulaciones que la rodean (5). Este se ve influenciado por una serie de factores tales como la ocupación, actividades diarias, edad, género y discapacidades físicas, atributos que se deben de tomar en cuenta para tener un confort en las estaciones de trabajo. Los rangos de movimientos se pueden utilizar como datos de referencia para los espacios de trabajo (6).

Para estimar los Roms en una articulación, se utiliza la técnica de goniometría; dicho término se deriva de los vocablos griegos: gonia, que significa “ángulo” y metron que significa “medidas”. Por lo que, a la goniometría se refiere a la medida de los àngulos, en particular aquellos creados en las articulaciones del cuerpo humano (7). Existen diferentes estudios acerca de los rangos de movimientos, mismos que se han enfocado en las diversas áreas de estudio previamente mencionadas; tal es el caso de la investigación llevada a cabo por (4), en la cual se analizó la relación entre los rangos de movimiento con las principales medidas utilizadas en un lugar de trabajo, afirmando que estos datos sirven de referencia para el diseño de equipo. A su vez, la investigación (8) se enfoca en una evaluación de rangos de movimientos posteriores a dos procedimientos quirúrgicos, para comparar cuál de los dos procedimientos tiene una menor reducción en los Rom cervicales.

Las consecuencias de no trabajar en un lugar adaptado a las características y necesidades físicas de los trabajadores, son el aumento de lesiones invalidantes ocasionadas por movimientos repetitivos y de lesiones musculo-esqueléticas, que pueden llegar a ser de gravedad, 
además de una multitud de padecimientos físicos crónicos en la espalda, las extremidades y el cuello, asociados a la mala postura del cuerpo (3).

Anteriormente, se han realizado investigaciones de los rangos de movimiento (ROM), con diversos enfoques, desde aplicaciones en el área del deporte hasta investigaciones médicas para determinar la eficiencia de técnicas de cirugía y medir la rehabilitación de lecciones, como en uno de los trabajos realizados que consistió en la implementación de un sistema para la evaluación de la articulación de la rodilla de flexión-extensión, con un sistema híbrido de medición angular, a través de un goniómetro (9).

Otro trabajo similar (10) evaluó la movilidad articular de la cadera y la rodilla en los movimientos fundamentales, a través de un goniómetro universal. Este trabajo fue realizado en una población de estudiantes de pedagogía en educación física en la Universidad Autónoma de Chile, con la finalidad de determinar si existía una diferencia significativa entre los rangos de movimiento de los hombres y las mujeres. Otros autores (11) determinaron rangos de movimiento para la evaluación y el diagnóstico del hombro, utilizando el sensor Kinect para medir los Rom del hombro.

\section{Materiales y métodos}

—ntre los materiales utilizados para esta investigación, se encuentra una hoja de consentimiento Ede elaboración propia, que tenía como fin para informar al participante sobre el objetivo del estudio y los posibles riesgos que tenía al participar en él, además de contar con un respaldo que autentificara el trabajo ante los sujetos de estudio; también se usó un formato de registro. Posteriormente, se utilizó el flexómetro del equipo antropométrico marca Rosscraft® para determinar la estatura de los participantes que conformaron la muestra y se empleó una báscula analógica marca Seca, para medir el peso de cada estudiante. Para la medición de los rangos de movimiento (ROM) fue empleado un inclinómetro de burbuja marca Baseline®, debido a que se caracteriza por su precisión para obtener grados de movimiento, sobre todo en espalda y extremidades superiores.

Se calculó el tamaño de la muestra para poblaciones finitas, tomando en cuenta la cantidad total de alumnos inscritos en la carrera de ingeniería industrial y de sistemas, la cual fue de 367 estudiantes. El tamaño de muestra se calculó por medio de la ecuación 1, y constó de de 50 alumnos con un margen de error de $5 \%$ y un nivel de confianza del $95 \%$.

$$
n=\frac{N Z^{2} p q}{d^{2}(N-1)+Z^{2} p q}
$$




\section{Donde:}

n: tamaño de la muestra.

N: tamaño de la población.

Z: valor de Z crítico, calculado en las tablas del área de la curva normal o nivel de confianza. d: Nivel de precisión absoluta.

p: proporción aproximada del fenómeno en el estudio en la población de referencia. q: proporción de la población de referencia que no presenta el fenómeno en estudio (1-p). La muestra fue tomada a conveniencia; una vez que fueron invitados los alumnos a participar en este estudio, se les pidió que leyeran y firmaran la hoja de consentimiento informado, al mismo tiempo que se le platicaba brevemente en lo que consistirían dichas las mediciones. Todos los participantes fueron informados del tiempo de duración de la medición, el cual fue de alrededor de 20 minutos por persona. Posteriormente, se procedió a medir la estatura del sujeto de prueba, por medio del kit antropométrico, indicándole al practicante que permaneciera erguido; se realizaron tres mediciones y fue registrada la mayor de ellas en la hoja de registro de datos; después, se pesó a los participantes.

Luego, se procedió a tomar los movimientos de las articulaciones del miembro superior (12), explicándoles a los participantes que todos los movimientos realizados debían llevarlos a cabo sin sentir molestia o dolor. Para los Rom del codo se colocó al participante en posición vertical, con los brazos a los lados y los codos completamente extendidos. Una vez posicionado el inclinómetro se registraron los grados de flexión y extensión de la articulación; este procedimiento se llevó a cabo tanto para el codo izquierdo como para el derecho. En el antebrazo, los Rom fueron determinados para los movimientos de pronación y supinación del mismo. Enseguida se procedió con la articulación del hombro, midiendo los grados de flexión, extensión, abducción y aducción en ambos hombros, colocando el inclinómetro en el brazo a la altura del bíceps. Por último, los Rom de la muñeca fueron medidos colocando la mano sobre una superficie plana, para obtener los grados de flexión, extensión, desviación radial y cubital. En la imagen 1, se puede apreciar la colocación del inclinómetro de burbuja para obtener los ángulos, así como el tipo de movimiento realizado para cada prueba. 


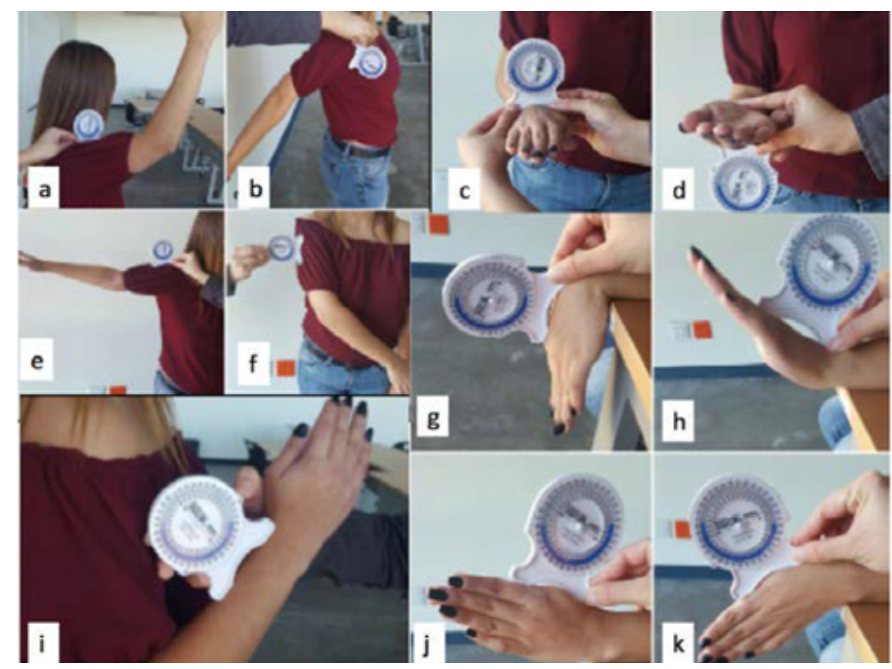

Imagen 1. Rangos de movimiento medidos en miembro superior

a) Hombro en flexión. b) Hombro en extensión. c) Pronación de antebrazo. d) Supinación de antebrazo. e) Hombro en abducción. f) Hombro en aducción. g) Muñeca en flexión. h) Muñeca en extensión. i) Codo en flexión. j)Muñeca en desviación radial. k) Muñeca en desviación cubital.

\section{Aspectos éticos}

$\mathrm{S}$ egún la Resolución 8430 de 1993 del Ministerio de Salud de Colombia, la presente investigación fue clasificada con riesgo mínimo.

\section{Resultados}

T a muestra obtenida fue de 50 alumnos, de los cuales 26 fueron hombres y 24 mujeres, Lcon una edad de 25,24 \pm 4,545 y 24,477 \pm 4,759 años, respectivamente. En la tabla 1 se puede observar que el Índice de Masa Corporal (IMC) en los varones tuvo una media de 25,24, mientras que en las mujeres fue de 24,477. Teniendo en cuenta lo propuesto por la Organización Mundial de la Salud (oms) (13), los hombres de la muestra padecen obesidad, ya que el Imc es superior a 25, mientras que las mujeres se encuentran en su peso normal.

Tabla 1. Datos demográficos de la muestra

\begin{tabular}{lcccccc}
\hline \multirow{2}{*}{ Datos demográficos } & \multicolumn{2}{c}{$\begin{array}{c}\text { Muestra Total } \\
\mathbf{N = 5 0}\end{array}$} & \multicolumn{2}{c}{$\begin{array}{c}\text { Mujeres } \\
\mathbf{N = 2 4}\end{array}$} & \multicolumn{2}{c}{$\begin{array}{c}\text { Hombres } \\
\mathbf{N = 2 6}\end{array}$} \\
\cline { 2 - 7 } & $\overline{\mathrm{X}}$ & $\Sigma$ & $\overline{\mathrm{x}}$ & $\sigma$ & $\overline{\mathrm{x}}$ & $\sigma$ \\
\hline Edad (años) & 21,12 & 1,71 & 21,208 & 1,641 & 21,038 & 1,8 \\
\hline Estatura (mts) & 1,7012 & 0,1084 & 1,6112 & 0,0626 & 1,7842 & 0,0669 \\
\hline Peso (kg) & 72,36 & 17,13 & 63,28 & 11,1 & 80,73 & 17,61 \\
\hline IMc & 24,874 & 4,617 & 24,477 & 4,759 & 25,24 & 4,545 \\
\hline
\end{tabular}


Los resultados de los Rom del codo y el antebrazo se muestran en la tabla 2, en la que se puede observar que la flexión de la muestra total tuvo mayor movilidad que el derecho con una media de $129,04^{\circ} \pm 27,03$, mientras que la pronación y supinación del lado derecho fue mayor, con una media de $92,86^{\circ} \pm 12,06$ y $91,46^{\circ} \pm 7,22$ de supinación. Por otra parte, las mujeres presentaron mayor movilidad que los hombres en los movimientos realizados con el miembro derecho y en supinación izquierda.

Tabla 2. ROM para articulaciones del miembro superior

\begin{tabular}{|c|c|c|c|c|c|c|c|}
\hline \multirow{2}{*}{$\begin{array}{c}\text { Movimiento } \\
\mu \text { (grados) }\end{array}$} & & \multicolumn{2}{|c|}{ Muestra Total } & \multicolumn{2}{|c|}{ Mujeres } & \multicolumn{2}{|c|}{ Hombres } \\
\hline & & $\sigma$ & $\mu$ (grados) & $\sigma$ & $\mu$ (grados) & $\sigma$ & \\
\hline \multicolumn{8}{|c|}{ Codo y antebrazo } \\
\hline \multirow{2}{*}{ Flexión } & (D) & 123,8 & 27 & 128,33 & 20,62 & 119,62 & 31,62 \\
\hline & (I) & 129,04 & 27,03 & 126 & 28,23 & 131,85 & 26,1 \\
\hline \multirow{2}{*}{ Pronación } & (D) & 92,86 & 12,06 & 93,88 & 15,81 & 91,92 & 7,63 \\
\hline & (I) & 88,94 & 7,15 & 90,92 & 5,57 & 87,12 & 8,02 \\
\hline \multirow{2}{*}{ Supinación } & (D) & 91,46 & 7,22 & 93,46 & 7,44 & 89,62 & 6,62 \\
\hline & (I) & 90,76 & 12,21 & 89,38 & 15,83 & 92,04 & 7,62 \\
\hline \multicolumn{8}{|c|}{ Hombro } \\
\hline \multirow{2}{*}{ Flexión } & (D) & 105,52 & 22,62 & 100,67 & 20,64 & 110 & 23,83 \\
\hline & (I) & 108,44 & 20,73 & 108 & 17,85 & 108,85 & 23,42 \\
\hline \multirow{2}{*}{ Extensión } & (D) & 42,8 & 9,48 & 43,75 & 10,86 & 41,92 & 8,13 \\
\hline & (I) & 39,86 & 11,43 & 38,54 & 12,72 & 41,08 & 10,19 \\
\hline \multirow{2}{*}{ Abducción } & (D) & 88,7 & 18,51 & 82,92 & 14,66 & 94,04 & 20,3 \\
\hline & (I) & 86,5 & 20,58 & 85,42 & 16,01 & 87,5 & 24,34 \\
\hline \multirow{2}{*}{ Aducción } & (D) & 14,25 & 7,51 & 13,83 & 9,23 & 14,62 & 5,64 \\
\hline & (I) & 13,66 & 8,81 & 14,38 & 11,64 & 13 & 5,14 \\
\hline \multicolumn{8}{|c|}{ Muñeca } \\
\hline \multirow{2}{*}{ Flexión } & (D) & 70,7 & 12,48 & 71,88 & 13,14 & 69,62 & 11,99 \\
\hline & (I) & 82,9 & 102,2 & 68,75 & 14,39 & 96 & 141,2 \\
\hline \multirow{2}{*}{ Extensión } & (D) & 54,18 & 12,07 & 51,83 & 11,62 & 56,35 & 12,29 \\
\hline & (I) & 56,58 & 11,52 & 54,17 & 11 & 58,81 & 11,74 \\
\hline \multirow{2}{*}{ Desviación radial } & (D) & 42,64 & 14,84 & 40,29 & 14,47 & 44,81 & 15,13 \\
\hline & (I) & 40,44 & 10,54 & 37,5 & 10,43 & 43,15 & 10,09 \\
\hline \multirow{2}{*}{ Desviación cubital } & (D) & 42,82 & 11,83 & 43,67 & 10,72 & 42,04 & 12,93 \\
\hline & (I) & 39,44 & 10,92 & 39,25 & 12,16 & 39,62 & 9,89 \\
\hline
\end{tabular}


En la tabla 3 se muestran los resultados obtenidos después de realizar una prueba T para dos muestras, la cual se llevó a cabo con un nivel de confianza de $95 \%$, para determinar la existencia de diferencia significativa entre los rom de las mujeres y de los hombres. Se obtuvo el p-value y el intervalo de diferencias para cada medición a través del software Minitab 17®, a partir del cual se pudo apreciar que en la abducción derecha del hombro el p-value es de 0,032 , y su intervalo de diferencia no incluye al cero, por lo que se rechaza $\mathrm{H}_{0}$, indicando que existe diferencia significativa entre los hombres y las mujeres en este movimiento. En el resto de los rangos de movimiento no se encontró diferencia significativa, ya que todos los p-values arrojados en el análisis estadístico fueron mayores al nivel de significancia, además de que los intervalos para la diferencia contienen al cero.

Tabla 3. Resultados de las pruebas T para la diferencia de medias

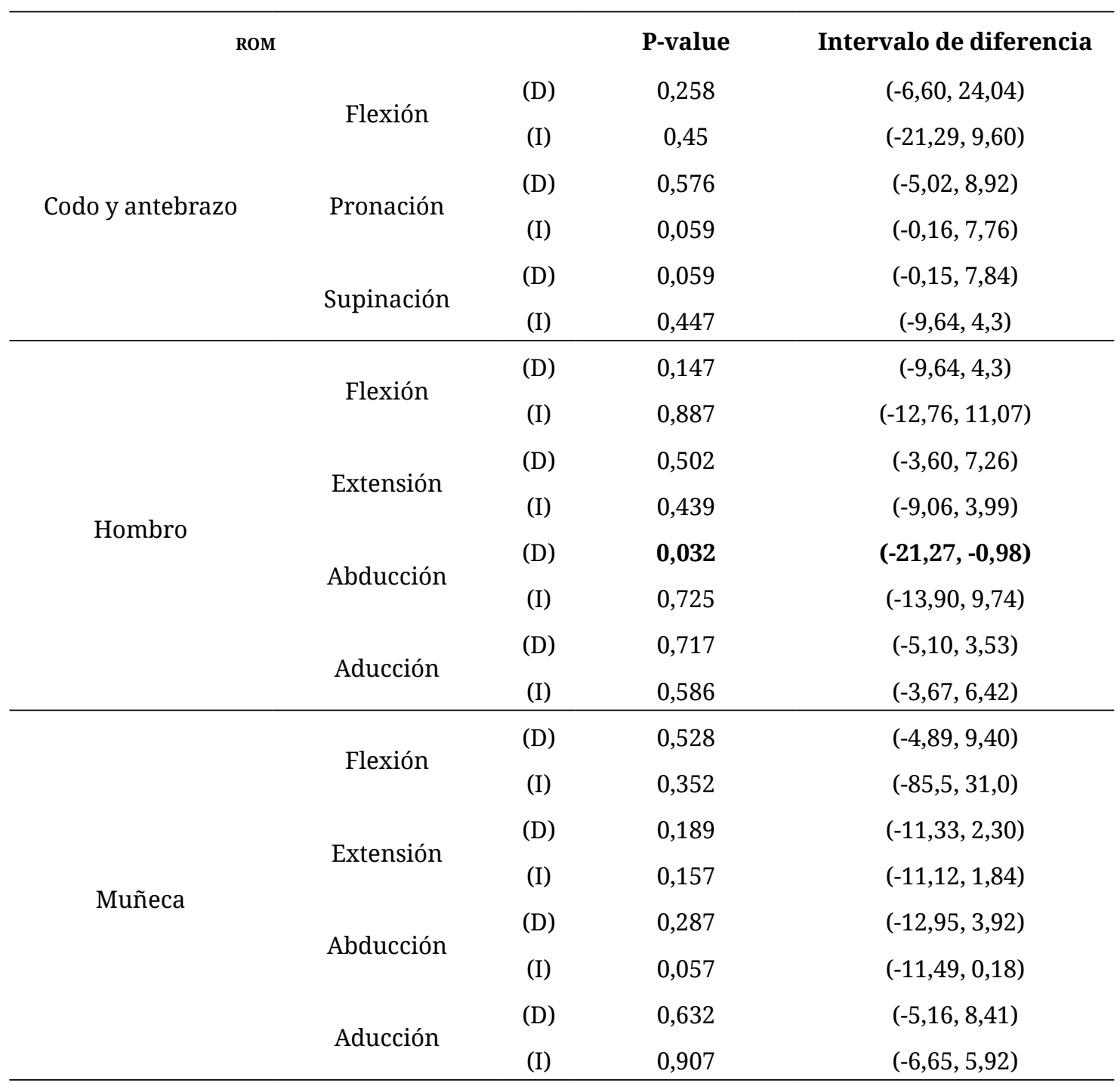




\section{Discusión}

Con el uso del inclinómetro de burbuja y la correcta ubicación del mismo en las articulaciones móviles principales del miembro superior, se logró conocer el rango máximo de movimiento del miembro superior en sus principales movimientos. La prueba de T efectuada en el estudio arrojó como resultado que solamente existe una diferencia significativa en los Rom entre hombres y mujeres en la abducción derecha del hombro, con un p-value de 0,032. En el resto de los movimientos no se encontró evidencia de diferencia significativa.

La recopilación de estos datos es importante para el diseño de estaciones y lugares de trabajo, para que éstas puedan ser adaptadas a los usuarios tomando en cuenta sus limitaciones de movimiento. De esta forma, se ayuda a prevenir las lesiones músculo-esqueléticas relacionadas al trabajo y las enfermedades laborales que puedan ser generadas por movimientos y posturas forzadas adoptadas por los trabajadores, debido al diseño de las instalaciones de trabajo; además, puede tener como beneficio un lugar de trabajo propicio para las distintas capacidades del usuario, que cumpla con los requerimientos de ergonomía laboral adecuados.

Para futuras investigaciones se aconseja determinar los Rom a través de software especializado, tales como el Optitrack ${ }^{\circledR}$ y kinovea ${ }^{\circledR}$, lo cual ayudaría a reducir el error humano o de igual manera se puede realizar un comparativo de los diversos instrumentos de medición de los rangos de movimiento para medir la eficiencia de cada uno de ellos y así poder determinar cuál es mejor método.

Anteriormente, se ha resaltado a la ergonomía como una serie de acciones fundamentales para la prevención de la presencia de lesiones músculo-esqueléticas en los trabajadores, la cual se encarga de buscar el bienestar laboral y social, previniendo la aparición de cualquier riesgo dentro de este ámbito (14). Actualmente, entre sus retos se encuentra la prevención de desórdenes traumacumulativos relacionados al trabajo, que han sido generados por la industria, donde las lesiones músculo-esqueléticas se asocian a actividades que implican manipulación de carga, trabajo repetitivo y la aplicación de fuerzas por encima de las capacidades de los trabajadores (15).

Otros autores (16) mencionan que la mejora en productividad, rendimiento físico y prevención de enfermedades ocupacionales en una industria, se logra a través del diseño del lugar de trabajo adecuado a las habilidades de cada individuo, además de que se reduce la cantidad de errores, accidentes y tiempo de aprendizaje. Los beneficios que se tiene al trabajar en un lugar adecuado a las capacidades del usuario y que cumple con el medio ambiente laboral, son la prevención de enfermedades, la reducción de la fatiga física y mental, la disminución del estrés y el aumento en la calidad de vida de los trabajadores $(17,18)$. Por otra parte, la reducción de padecimientos asociados a malas posturas da como resultado una reducción en los costos operativos, ya que una persona incapacitada implica la contratación 
de otra para reemplazarla, o bien cubrir la posición con tiempos extra, a la vez que genera un descontrol en la distribución del personal en el área de trabajo.

Para futuras investigaciones se recomienda realizar este tipo de estudios con trabajadores administrativos, incluyendo otras categorías de edad, que permita comparar los rangos de movimiento y determinar la relación de los mismos con la presencia de lesiones músculo-esqueléticas. Además de ampliar la base de datos, permitiendo utilizar dicha información para el diseño de puestos de trabajo acordes a las características de la población de estudio.

\section{Agradecimientos}

C agradece a los estudiantes que formaron parte del estudio y a los prestadores de servicio social del Laboratorio de Ergonomía y Métodos, de la División Multidisciplinaria de Ciudad Universitaria UACJ, por el apoyo brindado durante el trabajo de campo.

\section{Descargos de responsabilidad}

\footnotetext{
工 os autores declaran no tener conflicto de intereses en la presente investigación.
}

\section{Referencias}

1. Balderrama C, Ariza S, Gaytán G, Soto L, Lom J, Moreno L, Martínez A. Ergonomía en el diseño gráfico e industrial. Juarez, Chihuahua: Universidad Autónoma de Ciudad Juárez; 2011.

2. Gutiérrez Henríquez M. Ergonomía e investigación en el sector salud. Cienc. enferm. [Internet]. 2014 dic [citado 2017 sep 19]; 20(3): 7-10. Disponible en: http://www.scielo. cl/scielo.php?script=sci_arttext\&pid=S071795532014000300001\&lng=es. http://dx.doi. org/10.4067/S0717-95532014000300001

3. Bascuas J, Hueso R. Ergonomía: 20 preguntas básicas para aplicar la ergonomía en la empresa. 2nd ed. Madrid: Constitución y leyes, S. A; 2012.

4. Fernández JE, Marley R, Noriega S, Ibarra G. Ergonomía ocupacional, diseño y adminitración del trabajo. México: International Journal of industrial Engineering; 2008.

5. Ibarra G, Fernández J, Ware B, Mital A, Marley R, Reyes S, Range of Motion of the Upper Extremity and Spine Joints En Mexican Adulds: A Pilot Study. International Conference on Industrial Engineering. 2010, oct 17. Mexico. Proceedings of the 15th Annual Conference on Industrial Engineering- Theory, Application and Practice, Mexico; 2010. p. 519-23. 
6. Hussain A, Case K, Marshall R, Summerskill S. Join mobility and inclusive challenges. IJIE. 2016; (53): 67-79.

7. Norkin C, White J. Measurement of Joint Motion. A guide to goniometry. Philadelphia: Davis Company; 2016.

8. Yue W, Liu X, Zhu X, Zhou R, Cui C, Li J. Postoperativethree-Dimensional Cervical Range of Motion and Neurological Outcomes in Patients with Cervical Ossification of the Posterior Longitudinal Ligament: Cervical Laminoplasty Versus Laminectomy with Fusion. Clin. Neurol. Neurosurgry. 2015; (134): p. 17-23.

9. Tognetti A, Carbonaro N, de Rossi D, Lurossi F. Wearable Goniometer and Accelerometer Sensory Fusion for Knee Joint Angle Measurement in Daily Life. Sensors. 2015 nov 11; 15(11), p. 28435-455; doi: 10.3390/s151128435

10. Godoy-Cumillaf A, Valdés-Badilla P, García A, Grandón M, Lagos Del Canto L, Aravena R, Herrera-Valenzuela T, Bruneau J, Durán S. Somatotipo y rangos de movilidad articular de cadera y rodilla en estudiantes universitarios. Nutr Hosp. 2015; (32), 2903-9 Disponible en: http://www.redalyc.org/articulo.oa?id=309243321073

11. Lee SH, Yoon C, Chung SG, Kim HC, Kwak Y, Park H-w, et al. Measurement of Shoulder Range of Motion in Patients with Adhesive Capsulitis Using a Kinect. PLoS ONE. 2015; 10(6). doi: https://doi.org/10.1371/journal.pone.0129398

12. Pheasant S. Bodyspace, Anthropometry, Ergonomics and the Design of Work. Philadelphia: Taylor \& Francis Inc; 2013.

13. Organizacion Mundial de la Salud. Obesidad y Sobrepeso [sitio virtual]; 2016 oct. Centro de prensa [citado 2016 oct 10]; [1 pantalla]. Disponible en: http://www.who.int/mediacentre/factsheets/fs311/es/

14. Garcia-Luna J. La ergonomía en la construcción de la salud de los trabajadores en Colombia. Rev Cienc Salud. 2014; 12, p. 77-82. doi: http://dx.doi.org/10.12804/revsalud12. esp. 2014.08

15. Hernández P. Principales brechas de la Ergonomía en América Latina: a quince años del siglo XXI. Rev Cienc Salud. 2016; 14, p. 5-10. doi: http://dx.doi.org/10.12804/revistas. urosario.edu.co/revsalud/vol14num02016

16. Rodríguez Y, Pérez E. Ergonomía y Simulación Aplicadas a la Industria. Red de Revistas Científicas de América Latina y el Caribe, España y Portugal [Redalyc]. 2011; 32, 2-11.

17. Díaz J, Vásquez N, Dolores M, Rojas M. Trabajo, Ergonomía y calidad de vida. Una aproximación conceptual e intregración. Salud de los trabajadores. 2007; 51-58. 